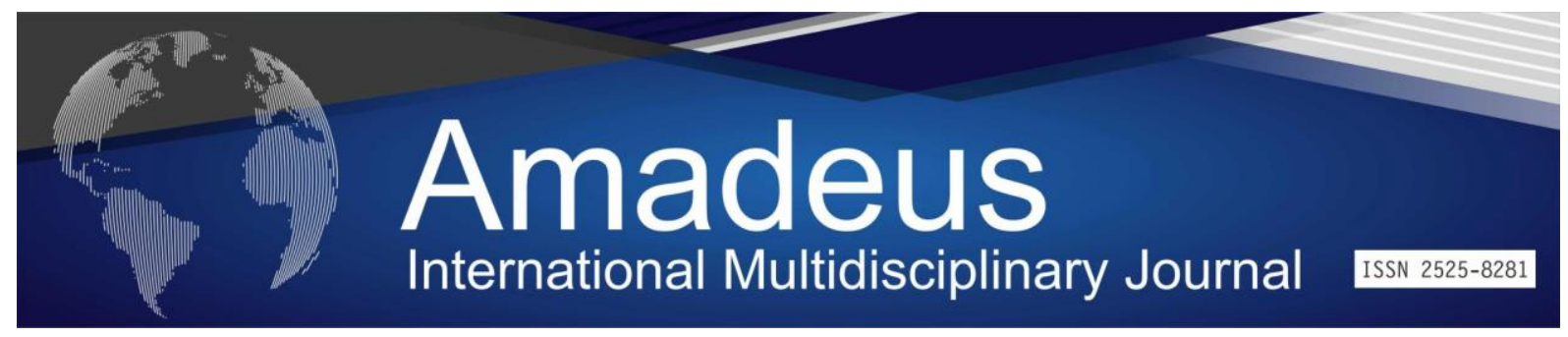

DOI: 10.14295/aimj.v5i9.140

\title{
Hemorrhagic Neurological Manifestations in a Patient With COVID - 19: Case Report
}

Ramille Gonçalves

Oliveiral,

Constantino Felipe

Leandro Clementino ${ }^{2}$, Priscila Teixeira Dias ${ }^{3}$, Lucas Tavares Cruz de Albuquerque $^{4}$, Marcus Vinicius Maia Passos Filho ${ }^{5}$,

Raphael Justo Cavalcante , $^{6}$ Raphael Xenofonte Morais Pinheiro $^{7}$
Abstract: SARS-CoV-2 infection has had a major impact on global health, affecting from the respiratory system to the central nervous system, generating a wide spectrum of clinical manifestations. Stroke has been reported in the recent literature when addressing the neurological repercussions in patients affected by COVID-19, with unfavorable outcomes when compared to those not infected by SARS-CoV-2. There are multiple theories that try to elucidate neural clinical manifestations, from hematogenous infection to direct entry through the cribiform blade. This article shows a case report of a patient with COVID-19 and neurological involvement with diagnostic hypotheses of acute necrotizing encephalopathy, a rare post-viral infection condition in adults and ischemic stroke with subsequent hemorrhagic transformation, correlating the aspects observed in the image and in the patient's clinic with the current literature and theories.

Keywords: Coronavirus infections, Acute necrotizing encephalopathy, Hemorrhagic transformation, Ischemic stroke.

\footnotetext{
1 Acadêmica de medicina da Faculdade de Medicina Estácio de Juazeiro do Norte (FMJ) - Ceará, Brasil. Contato: ramille.goncalves@hotmail.com;

2 Acadêmico de Medicina da Faculdade de Medicina Estácio de Juazeiro do Norte (FMJ), Ceará, Brasil. Contato: constantinoclementino1@gmail.com;

3 Acadêmica de medicina da Faculdade de Medicina Estácio de Juazeiro do Norte (FMJ) - Ceará, Brasil. Contato: priscila21.02@outlook.com;

4 Acadêmico de Medicina da Faculdade de Medicina Estácio de Juazeiro do Norte (FMJ), Ceará, Brasil. Contato: lucastavares47@gmail.com;

5 Acadêmica de medicina da Faculdade de Medicina Estácio de Juazeiro do Norte (FMJ) - Ceará, Brasil. Contato: mpassosf@hotmail.com;

${ }^{6}$ Neurorradiologista, Hospital São Camilo, Crato/CE. Contato: raphaelcory @ gmail.com;

${ }^{7}$ Radiologista docente da Faculdade de Medicina Estácio de Juazeiro do Norte (FMJ)- Ceará, Brasil. Contato: radioimip2011@gmail.com.
} 


\title{
Manifestações Neurológicas Hemorrágicas em Paciente com COVID - 19: Relato de Caso
}

\begin{abstract}
Resumo: A infeção pelo SARS-CoV-2 tem tido grande impacto na saúde mundial, afetando desde o sistema respiratório até o sistema nervoso central, gerando um amplo espectro de manifestações clínicas. $\mathrm{O}$ acidente vascular cerebral tem sido relatado na literatura recente quando abordado as repercussões neurológicas em pacientes acometidos pela COVID-19, tendo desfechos desfavoráveis quando comparados aqueles não infectados pelo SARS-CoV-2. São as múltiplas as teorias que tentam elucidar as manifestações clínicas neurais, desde a infeção por via hematogênica até a entrada direta pela lâmina cribiforme. Esse artigo mostra um relato de caso de um paciente com COVID-19 e acometimento neurológico com hipóteses diagnosticas de encefalopatia necrotizante aguda, uma condição de pós infecção viral rara em adultos e acidente vascular isquêmico com posterior transformação hemorrágica, correlacionando os aspectos observados na imagem e na clínica do paciente com a literatura vigente e as teorias.
\end{abstract}

Palavras-chave: Coronavirus infections, Acute necrotizing encephalopathy, Hemorrhagic transformation, Ischemic stroke.

\section{Introdução}

Um novo coronavírus, o SARS-CoV-2, causou um surto de pneumonia grave (COVID-19) na China que se espalhou rapidamente pelo mundo (Kandemirli et al, 2020). Evidências recentes destacam uma porcentagem relativamente alta (36\%) dos sintomas do sistema nervoso central, incluindo dor de cabeça, estado mental alterado, doença cerebrovascular aguda e epilepsia em pacientes com COVID-19, sendo que a taxa de sintomas neurológicos é maior em pacientes com doença respiratória mais grave (Mao et al, 2020). De fato, o SARS-CoV-2, pode infectar o sistema nervoso, músculo esquelético e trato respiratório, pelo fato de todos os sistemas compartilham os mesmos receptores DA enzima conversora de angiotensina ACE-2 presentes no sistema respiratório (Baig, 2020).

Foi observado um aumento significativo das taxas de AVC em pacientes com COVID19, com piores resultados em comparação com o grupo não COVID-19, um número significativamente menor de pacientes com um bom resultado, em virtude da COVID-19 provocar a liberação de citocinas pró-inflamatórias com efeito direto na ruptura da placa por inflamação local e ativação da coagulação (Benussi et al., 2020).

Como a COVID-19 afeta mais os idosos e aqueles com condições preexistentes, pacientes com condições neurológicas prévias e sintomas respiratórios agudos têm um risco 
aumentado de encefalopatia na apresentação inicial (Espinosa et al., 2020). A encefalopatia necrosante aguda (ENA) é uma condição médica caracterizada por lesões simétricas multifocais envolvendo o tálamo, tronco cerebral, cerebelo e substância branca (Kandemirli et al., 2020).

Apesar dos crescentes estudos e relatórios sobre a ENA nos últimos anos, a patogênese da doença permanece incerta ( $\mathrm{Li}$ et al., 2020). É geralmente a hipótese de que a "tempestade de citocinas" em resposta ao vírus induz inflamação sistêmica que pode causar a encefalopatia observada (Akins et al., 2020). A hipercitinemia resultante acredita-se que provoque várias respostas imunes, que causam dano celular endotelial, provocando alterações na permeabilidade do endotélio vascular e ruptura estrutural da barreira hematoencefálica (Ichiyama et al., 2020). Um estudo de Takeshita demonstrou que as lesões podem ser observadas usando-se ressonância magnética aprimorada mesmo antes dos sintomas neurológicos de ENA aparecem (Li et al., 2020).

Dessa forma, constata-se a relevância de iniciar a examinar as manifestações dos coronavírus devido ao seu incontestável neurotropismo (Arabi et al, 2020). Esse estudo, portanto, apresenta como finalidade expor um relato de caso de SARS-CoV-2 com comprometimento do sistema nervoso, através de uma abordagem voltada para os achados radiológicos, confrontando os elementos observados na imagem e clínica do enfermo com a bibliografia atual.

\section{Relato do Caso}

Paciente 41 anos, sexo masculino, admitido no dia 27/06/2020 com história de febre, diarreia e desconforto respiratório iniciados no dia 17/06/2020. Realizou sorologia que evidenciou IgG e IgM positivos para SARS-COV-2, com TC de tórax mostrando comprometimento pulmonar superior a $70 \%$, sendo portador de insuficiência renal crônica dialítica há 5 anos.

Evoluiu com insuficiência respiratória e intubação orotraqueal, com admissão em UTI, com piora do estado geral e queda do nível de consciência, com escala de coma de Glasgow de 3, sem resposta motora ou respiratória, com relato de pupilas fixas midiatricas, reflexo corneopalpebral e oculovestibular ausentes, sem reflexos de faringe/tranqueia. Realizada TC de Crânio em corte axional com resultados mostrados nas imagens abaixo. A observação de estado Clínico associado a imagens, foi decretado dano em tronco cerebral, sendo decretado 
protocolo para morte cerebral às 22:40 e repetido 12 horas. Evoluindo a óbito no dia 05/07/2020.
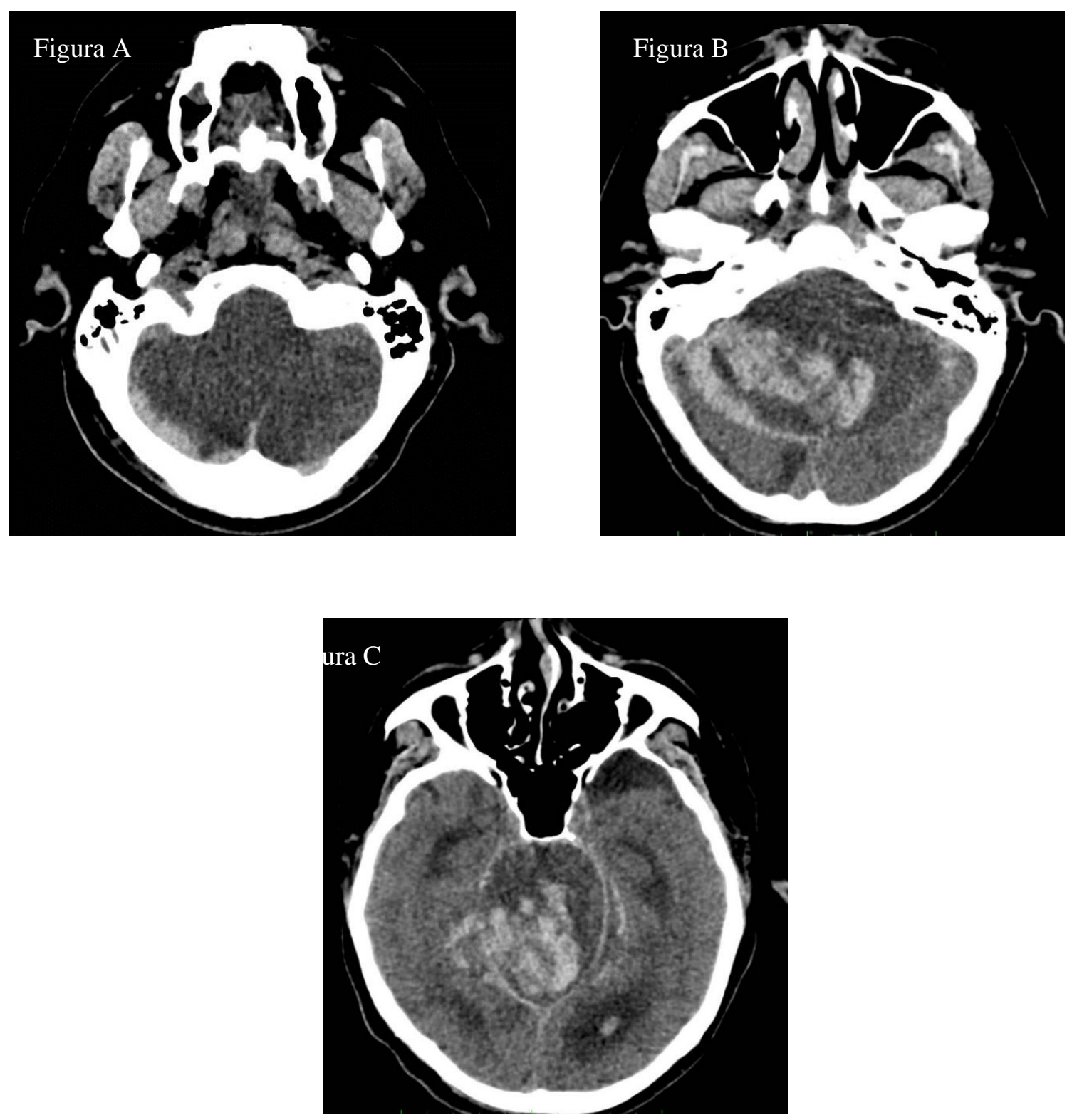

Figuras A, B e C: Extensa hipodensidade envolvendo todo o tronco cerebral - ponte, bulbo e mesencéfalo, bem como a substância branca e o córtex nos hemisférios cerebelares, associado a área de hemorragia no vêrmis cerebelar, determinando apagamento dos espaços liquóricos / cisternas na fossa posterior.

Nas imagens é tido como impressão diagnostica uma lesão hemorrágica extensa envolvendo o aspecto superior dos hemisférios cerebelares e vermis cerebelar. Associa-se ainda extensa hipodensidade corticossubcortical envolvendo o tronco cerebral e no hemiférios 
cerebelares, podendo estar relacionado a lesão isquêmica aguda / edema citotóxico. Pode ser notado ainda extensão do conteúdo hemorrágico para o interior dos ventrículos, notando maior quantidade de sangue no terceiro ventrículo.

\section{Discussão}

A infeção pelo SARS-CoV-2 vem se alastrando em todo o mundo, à medida que os índices dessa doença aumentam, as evidências de um acometimento cerebrovascular crescem significativamente, bem como outras formas de doença vascular (Ellul et al., 2020).

Pacientes que desenvolvem Síndrome da Angústia Respiratória Aguda (SARA) são classificados como graves e são mais predispostos a desenvolverem manifestações neurológicas, principalmente quadros cerebrovasculares agudos, distúrbios conscientes e lesão muscular esquelética (Mao et al., 2020).

O acometimento do tecido nervoso central pela COVID-19 tem sido elucidado em diversos estudos, a fim de esclarecer as múltiplas manifestações clínicas neurais causadas pelo vírus. A invasão provavelmente ocorre pela via hematogênica ou, preferencialmente, pela lâmina cribiforme e atinge o SNC a partir da via neuronal olfativa de maneira retrógrada, em semelhança ao vírus da Síndrome Respiratória Aguda Grave, gerando lesão tecidual, provavelmente, por dois mecanismos, sendo eles: lesão cerebral hipóxica e dano imunológico imunomediado (Ahmad \& Azam, 2020).

O paciente apresentou evolução grave do quadro cíinico viral com manifestações pulmonares e extrapulmonares, onde houve o acometimento de mais de $70 \%$ do pulmão e lesões hemorrágicas acometendo o tronco encefálico e cerebelo, com um importante edema perilesional, associado à hidrocefalia supratentorial, evoluindo de um estado comatoso ao desfecho fatal. Esse quadro clínico é semelhante a uma condição médica rara relatada na literatura, chamada encefalopatia necrosante aguda. Sua apresentação é variável, indo desde paralisia de nervos cranianos a deterioração súbita do estado mental em um curto espaço de tempo após uma infecção de etiologia viral.

O estudo de imagem se caracteriza por lesões simétricas multifocais em tálamo, tronco encefálico, substância branca cerebral e cerebelo (Poyiadji, et al., 2020). O distúrbio orgânico atrelado a esse diagnostico, quando evidenciado lesão pulmonar aguda, insuficiência hepática e/ou choque e edema cerebral generalizado geralmente ditam um pior prognostico, indo de encontro a evolução clínica do nosso paciente após a constatação da infeção por COVID-19 
(Li, et al., 2020). Entretanto, o predomínio dessa condição é relatado na faixa etária infantil, mas já existindo casos presuntivos relatados em adultos (Erasmo, et al., 2020).

Ademais, outra hipótese de forte suspeita é o acidente vascular isquêmico com transformação hemorrágica, a qual possui uma gênese de múltiplos fatores e fisiopatologia complexa (Sussman \& Connolly, 2013), e, tem incidência em aproximadamente $43 \%$ dos casos de acidente vascular cerebral isquêmico (Heit, Iv, \& Wintermark, 2017).

A presente patologia ocorreu em 3 dos 22 pacientes do estudo de Sweid et al. (2020) diagnosticados com SARS-CoV-2. A conversão hemorrágica adveio, principalmente, pelo uso de anticoagulação nos pacientes que apresentam elevados níveis de D-dímero, e todos necessitaram de descompressão cirúrgica, além disso, os mesmos apresentaram altos níveis de interleucina-6. Já no estudo de Reddy et al. (2020), apenas 1 dos 10 pacientes que tiveram AVC isquêmico apresentou a conversão hemorrágica. Com isso, pela falta de estudos com grande amostragem não se pode definir com maior precisão a incidência da conversão em pacientes com COVID-19.

Ambas teorias para definição do caso possuem fortes dados que explicam a evolução do paciente, porém a resposta ainda continua incerta em meio ao cenário mundial de saúde causado pela COVID-19.

\section{Conclusão}

Apesar de ser uma doença nova, a COVID-19 parece comprometer as formas graves pulmonares com acometimento encefálico associado, seja por fenômeno de tromboembolismo, sendo incerta ainda a natureza de tais eventos hipoxico-isquêmicos, bem como desfecho com encefalopatia necrotizante.

\section{Referências}

Ackermann, M., Verleden, S. E., Kuehnel, M., Haverich, A., Welte, T., Laenger, F., Vanstapel, A., Werlein, C., Stark, H., Tzankov, A., Li, W. W., Li, V. W., Mentzer, S. J., \& Jonigk, D. (2020). Pulmonary Vascular Endothelialitis, Thrombosis, and Angiogenesis in Covid-19. New England Journal of Medicine. DOI: https://doi.org/10.1056/nejmoa2015432

Akçai, S., Ozlu, T., Yilmaz, A. (2020). Radiological approaches to COVID-19 pneumonia. Turkish Journal of Medical Sciences, 50(3), 604-610. DOI: 10.3906/sag-2004-160 
Akin, P. T., Belko, J., Uyeki, T. M., Axelrod, Y., Lee, K. K., \& Silvertthorn, J. (2020). H1N1 Encephalitis Malignant Edema and Review of Neurological Complications of Influenza. Disponível: https://link.springer.com/article/10.1007\%2Fs12028-010-9436-0. Acesso em: 04 ago. 2020.

Arabi, Y. M. et al. (2020). Severe Neurologic Syndrome Associed With Middle East Respiratory Syndrome Corona Virus(MERS-CoV). 20 jan 2020. Springer Science and Business Media LLC. Disponível em: https://link.springer.com/article/10.1007\%2Fs15010015-0720-y. Acesso em: 06 de jun 2020.

Baig, A. M. (2020). Neurological Manifestations in COVID-19 Caused by SARS-CoV-2. Cns Neuroscience \& Therapeutics. Disponível em http://dx.doi.org/10.1111/cns.13372. Acesso em: 09 jun. 2020

Benussi, A. et al. (2020) Clinical Characteristics and Outcomes of Inpatients With Neurologic Disease and COVID-19 in Brescia, Lombardy, Italy. Neurology. Ovid Technologies (Wolters Kluwer Health). Disponível em: https://n.neurology.org/content/early/2020/05/22/WNL.0000000000009848.abstract. Acesso em 09 jun. 2020

Espinosa, P. S. et al (2020). Neurological Complications of Coronavirus Disease (COVID 19): Encephalopathy, BrainMRI and Cerebrospinal Fluid Fidings: Case 2. Dipsonível em: https://pesquisa.bvsalud.org/global-literature-on-novel-coronavirus-2019ncov/resource/en/covidwhomdl-32499974. Acesso em 04 ago. 2020.

Gao, L., \& Zhang, J. (2020). Pulmonary High-Resolution Computed Tomography (HRCT) Findings of Patients with Early-Stage Coronavirus Disease 2019 (COVID-19) in Hangzhou, China. Medical Science Monitor: International Medical Journal of Experimental and Clinical Research, 26, e923885-1

Inchiyama, T. et al (2020). Serum Cytokine Concentrations of Influenza-Associated Acute Necrotizing Encephalopathy. Pediatrics Iternational. Disponívem em: https://onlinelibrary.wiley.com/doi/abs/10.1111/j.1442-200X.2003.01822

x?sid=nlm\%3Apubmed. Acesso em: 04 ago. 2020

Jiang, X., Yin, Z., Wang, T., Zhai, N., Lu, F., Zhan, C., Han, Q. e Feng, C. (2020). COVID19 Desempenho da Tomografia Computadorizada Dinâmica (CT) e Observação de Alguns Indicadores Laboratoriais. Medical science monitor: revista médica internacional de pesquisas experimentais e clínicas, 26, e924403. DOI: 10.12659 / MSM.924403.

Kandemirli, S. L. et al (2020). MRI Findings in Patients in the Intensive Care Unit with COVID-19 Infection. Radiology. Disponível em: https://pubs.rsna.org/doi/10.1148/radiol.2020201697. Acesso em 09. Jun 2020

$\mathrm{Li}, \mathrm{H}$. et al (2020). Use of Magnetic Resonance in the Diagnosis and Prognosis of Acute Necrotizing Encephalopathy in a Chinese Teenager. Medicine, 05 jul. 2020. Disponível em: https://pubmed.ncbi.nlm.nih.gov/31689857/. Acesso em: 04 ago. 2020. 
Mao, L. et al (2020). Neurologic Manifestations of Hospitalized Patients With Coronavirus Disease 2019 in Wuhan,China. Jamaneurology. Dipsonível em https://jamanetwork.com/journals/jamaneurology/fullarticle/2764549. Acesso em 09 jun 2020.

McGonagle, D., O’Donnell, J. S., Sharif, K., Emery, P., Bridgewood, C. (2020). Immune mechanisms of pulmonary intravascular coagulopathy in COVID-19 pneumonia. Lancet Rheumatol, 1-9. DOI: 10.1016/S2665-9913(20)30121-1

Phend, C. (2020). COVID-19 Pulmonary Embolism Often Seen Outside the ICU. Medpage Today. Acesso em 25 de maio de 2020 de https://www.medpagetoday.com/infectiousdisease/covid19/86535.

Poyiadji, N., Cormier, P., Patel, P. Y., Hadied, M. O., Bhargava, P., Khanna, K., Nadig, J., Keimig, T., Spizarny, D., Reeser, N., Klochko, C., Peterson, L., \& Song, T. (2020). Acute Pulmonary Embolism and COVID-19. Radiology, 201955.

Wang, K., Kang, S., Tian, R., Zhang, X., Zhang, X., \& Wang, Y. (2020). Imaging manifestations and diagnostic value of chest CT of coronavirus disease 2019 (COVID-19) in the Xiaogan area. Clinical radiology, 75(5), 341-347. DOI: 10.1016/j.crad.2020.03.004

Yoon, S. H., Lee, K. H., Kim, J. Y., Lee, Y. K., Ko, H., Kim, K. H., Park, C. M., \& Kim, Y.H. (2020). Chest Radiographic and CT Findings of the 2019 Novel Coronavirus Disease (COVID-19): Analysis of Nine Patients Treated in Korea. Korean Journal of Radiology, 21(4), 494. DOI: https://doi.org/10.3348/kjr.2020.0132

Yuan, M., Yin, W., Tao, Z., Tan, W. e Hu, Y. (2020). Associação de achados radiológicos com mortalidade de pacientes infectados com o novo coronavírus de 2019 em Wuhan, China. PloS one , 15 (3), e0230548. DOI: 10.1371 / journal.pone.0230548.

\section{How to cite this article (APA format):}

Oliveira, Ramille Gonçalves; Clementino Constantino Felipe Leandro; Dias, Priscila Teixeira; Albuquerque, Lucas Tavares Cruz de; Passos Filho, Marcus Vinicius Maia; Cavalcante, Raphael Justo; Pinheiro, Raphael Xenofonte Morais (2020). Hemorrhagic Neurological Manifestations in a Patient With COVID - 19: Case Report. Am. In. Mult. J., Jul to Oct. (9) 5, 177-184.

Received: 09/04/2020;

Accepted: 09/08/2020. 